\title{
An atomic theory with no prime models
}

\author{
Tarek Sayed Ahmed \\ Department of Mathematics, Faculty of Science, \\ Cairo University, Giza, Egypt. \\ rutahmed@gmail.com \\ Received by Greg Restall \\ Published November 19, 2007 \\ http://www.philosophy.unimelb.edu.au/ajl/2007 \\ (C) 2007 Tarek Sayed Ahmed
}

Abstract: We construct an atomic uncountable theory with no prime models. This contrasts with the countable case.

We follow the standard textbook [I]. We start with the basic definitions to set up the notation. Let $\mathfrak{L}$ be a first order language, and T be a complete theory in $\mathfrak{L}$. A formula $\phi\left(x_{0}, \ldots x_{n-1}\right)$ is complete in $T$ if for all $\psi\left(x_{0}, \ldots x_{n-1}\right)$, we have

$$
\mathrm{T} \models \phi \rightarrow \psi \text { or } \mathrm{T} \models \phi \rightarrow \neg \psi \text {. }
$$

A formula $\theta\left(x_{0}, \ldots x_{n-1}\right)$ is completable in $T$ iff there is a complete formula $\phi\left(x_{0}, \ldots x_{n-1}\right)$ with $T \models \phi \rightarrow \theta$. A theory $T$ is atomic if every $\mathfrak{L}$ formula which is consistent with $T$ is completable in $T$. An $\mathfrak{L}$-structure $\mathfrak{M}$ is said to be atomic, if every $n$-tuple $a_{0}, \ldots a_{n-1} \in M$ satisfies a complete formula in $\mathrm{Th}(\mathfrak{M})$. A model $\mathfrak{M}$ is a prime model if it embeds elementarily in any model of $\operatorname{Th}(\mathfrak{M})$. A Theorem of Vaught [I] 2.3.2 says that If $\mathrm{T}$ is a complete countable theory, then $\mathrm{T}$ has a countable atomic - equivalently a prime model - if and only if it is atomic. The proof in the countable case appeals to the HenkinOrey omitting types theorem which we recall. Let $\Sigma$ be a set of formulas. T locally realizes $\Sigma$ if there is a formula $\phi\left(x_{0}, \ldots x_{n-1}\right)$ in $\mathfrak{L}$ such that

(i) $\phi$ is consistent with $\mathrm{T}$

(ii) For all $\sigma \in \Sigma, T \models \phi \rightarrow \sigma$.

T locally omits $\Sigma$ iff $\mathrm{T}$ does not locally realize $\Sigma$. Thus $\mathrm{T}$ locally omits $\Sigma$ if if and only if for any formula $\phi\left(x_{0} \ldots x_{n-1}\right)$ which is consistent with $T$, there exists $\sigma \in \Sigma$ such that $\phi \wedge \neg \sigma$ is consistent with T. M omits $\Sigma$ if no tuple in $\mathfrak{M}$ realizes all formulas in $\Sigma$. The Omitting Types Theorem [I] 2.2.15. states that 
THEOREM I (Henkin-Orey) Let T be a consistent theory in a countable language $\mathfrak{L}$, and for all $i<\omega$, let $\Sigma_{i}\left(x_{1} \ldots x_{n}\right)$ be a set of formulas in $n_{i}$ variables. If $\mathrm{T}$ locally omits each $\Sigma_{i}$, then $T$ has a model which omits each $\Sigma_{i}$.

Now the idea of Vaught's Theorem is follows. Let T be an atomic theory. For each $n<\omega$, let $\Sigma_{n}$ be the negation of complete formulas $\psi\left(x_{0} \ldots x_{n-1}\right)$ in $T$. Then T locally omits each set $\Sigma_{n}$. A model omitting all $\Sigma_{n}$ will be the desired model [I] 2.3.5. We show that the non-trivial implication ( $T$ is atomic implies $\mathrm{T}$ has a prime model) does not hold for uncountable languages of any greater power. The question as to wether Vaught's Theorem extends to the uncountable case, is natural, however to the best of our knowledge it was not dealt with in the literature. (It is proved though that atomic theories of size $\leqslant \mathbf{x}_{1}$ do have atomic models [4] and [3].) For a language $\mathfrak{L}, \operatorname{Md}_{\mathfrak{L}}$ denote the class of $\mathfrak{L}$ structures and $\mathfrak{F}_{\mathrm{L}}$ denotes the set of $\mathfrak{L}$ formulas.

Proof: Let $\mathfrak{T}^{\prime}=\left(\mathrm{T}^{0},<^{0}\right)$ be a normal Aronszajn tree.i.e,

(I) $\mathfrak{T}^{\prime 0}$ is a poset, and

(2) for every $a \in T^{0}, T^{0} \mid a=\left\{x \in T^{0}: x<^{0} a\right\}$ is well ordered by $<^{0}$. If for any ordinal $\beta$

$$
T_{\beta}^{0}=\left\{a \in T^{0}: \text { the order type of }\left(T^{0}\left|a, T^{0}\right| a \mid<^{0}\right)=\beta\right\} .
$$

Then the following hold:

(3) $\left|\mathrm{T}_{0}^{0}\right|=1$.

(4) There are no ordered subsets of $\mathfrak{T}^{\prime} 0$ of order type $\omega_{1}$.

(5) For every $\beta<\omega_{1},\left|T_{\beta}^{0}\right| \leqslant \boldsymbol{x}_{0}$.

(6) If $\beta<\omega_{1}$ is a limit ordinal, $a, b \in T_{\beta}^{0}$ and $\mathrm{T}^{0}\left|a=\mathrm{T}^{0}\right| \mathrm{b}$, then $\mathrm{a}=\mathrm{b}$.

(7) Each $a \in T^{0}$ has $\aleph_{0}$ immediate successors.

(8) If $\beta<\mu<\omega_{1}$ and $x \in T_{\beta}^{0}$ then there is a $y \in T_{\mu}^{0}$ such that $x<^{0} y$. (It is well known that in ZFC normal Aronszajn trees exist.)

Let $\mathfrak{L}$ be a first order language with one binary relation symbol $<$ and $\boldsymbol{N}_{1}$ unary relation symbols $\mathrm{T}_{\beta}\left(\beta<\omega_{1}\right)$. Let $\mathfrak{T}^{0}=\left(\mathfrak{T}^{\prime}, \mathrm{T}_{\beta}^{0}\right)_{\beta<\omega_{1}} \in \mathrm{Md}_{\mathfrak{L}}$. Let $\Sigma=\operatorname{Th}\left(\mathfrak{T}^{\prime 0}\right)$, the first order theory of $\mathfrak{T}^{0}$. Then $\Sigma$ is complete. Define $\mathfrak{T}^{1}$ as follows

$$
\begin{gathered}
T^{1}=\left\{f: f \text { is a function from } \beta<\omega_{1} \text { into } \omega\right. \\
\text { with } \left.|\{\beta: f(\beta)>0\}|<\boldsymbol{x}_{0}\right\} .
\end{gathered}
$$


Let $\mathfrak{T}^{\prime 1}=\left(\mathrm{T}^{1},<_{1}\right)$ where

$$
f<^{1} g \text { iff } f \subseteq g .
$$

For $\beta<\omega_{1}$, define $T_{\beta}^{1}$ as follows :

$$
T_{\beta}^{1}=\left\{f \in T^{1}: \operatorname{Dom}(f)=\beta\right\} .
$$

Now let

$$
\mathfrak{T}^{1}=\left(\mathfrak{T}^{\prime 1}, \boldsymbol{T}_{\beta}^{1}\right)_{\beta<\omega_{1}}
$$

We now prove

(9) $\mathfrak{T}^{1} \models \Sigma$ so that $\mathfrak{T}^{0} \equiv \mathfrak{T}^{1}$.

(Io) $\Sigma$ is atomic.

(II) Every elementary submodel of $\mathfrak{T}^{0}$ satisfies (4) but no elementary submodel of $\mathfrak{T}^{1}$ satisfy (4). This will prove our result (because a prime model would be elementary embeddable in $\mathfrak{T}^{0}$ and $\mathfrak{T}^{1}$.)

By (2), (3) and (6), $\mathfrak{T}^{\prime 0}$ is a meet semilattice. It is also easy to see that $\mathfrak{T}^{\prime 1}$ is a meet semilattice. We shall work in a definitional expansion of $(\mathfrak{L}, \Sigma)$ with a binary relation . with definition

$$
\begin{gathered}
\left(\forall v_{0} \forall v_{1} \forall v_{2}\right)\left(v_{2}=v_{0} \cdot v_{1} \longleftrightarrow v_{2} \leqslant v_{0} \wedge v_{2} \leqslant v_{1} \wedge\right. \\
\left.\left.\forall v_{3}\right)\left(v_{3} \leqslant v_{0} \wedge v_{3} \leqslant v_{1} \longrightarrow v_{3} \leqslant v_{2}\right)\right) .
\end{gathered}
$$

(I2) $\mathfrak{T}^{1}$ satisfies $(1)-(3)$ and (5) - (8).

For $k, m \in \omega$ and $\beta \in \omega_{1}$, let $\phi^{k, m, \beta} \in \mathfrak{F}_{\mathfrak{L}}$ be defined as $\mathrm{T}_{\beta}\left(v_{\mathrm{k}} \cdot v_{\mathrm{m}}\right)$. For $n \in \omega, i<2$ and $q \in{ }^{n} T^{i}$ define

$$
\Gamma_{\mathrm{q}}=\left\{\phi^{\mathrm{k}, \mathrm{m}, \beta}: \mathrm{k}, \mathrm{m}<\mathrm{n} \text {, and } \mathfrak{T}^{\mathrm{i}} \models \phi^{\mathrm{k}, \mathrm{m}, \beta}[\mathrm{q}]\right\} .
$$

For any $\mathrm{q} \in{ }^{n} T^{i}$, let $\phi_{\mathrm{q}}=\bigwedge \Gamma_{\mathrm{q}}$. For $i, j<2, n \in \omega, \mathrm{q} \in{ }^{n} \mathrm{~T}^{i}$ and $r \in{ }^{n} T^{j}$ define

$$
\mathrm{qI}_{\mathrm{n}} \mathrm{r} \text { iff } \models \phi_{\mathrm{q}} \longleftrightarrow \phi_{\mathrm{r}}
$$

By (7) (8) and (12) we have

(13) if $q \in{ }^{n} T^{i}$ and $r \in{ }^{n} T^{j}, q I_{n} r$ and $a \in T^{i}$, then there is $a b \in T^{j}$ such that

$$
\left(q_{0}, \ldots q_{n-1}, a\right) I_{n+1}\left(r_{0} \ldots r_{n-1}, b\right)
$$

Using (13) for $i \neq j$ (9) follows. By (13) again, for $i=j=0$, for every $\mathrm{q} \in{ }^{n} T^{0}, \phi_{q}$ is an atomic formula. This proves (10). Now we prove (II). Trivially every elementary submodel of $\mathfrak{T}^{0}$ satisfies (I)-(8). Let

$$
\mathfrak{T}^{*}=\left(\mathrm{T}^{*},<^{*}, \mathrm{~T}_{\beta}^{*}\right)_{\beta<\omega_{1}}
$$


be an elementary submodel of $\mathfrak{T}^{1}$. For any $\beta<\omega_{1}$, choose $f_{\beta} \in T_{\beta}^{*}$. Define $\mathrm{H}: \omega_{1} \rightarrow \omega_{1}$ by

$$
\begin{gathered}
H(\beta)=\text { the greatest } \mu \text { such that } f_{\beta}(\mu) \neq 0 \text {, if such } \mu \text { exists } \\
=0 \text { otherwise } .
\end{gathered}
$$

$H$ is well defined since $\left\{\mu: f_{\beta}(\mu) \neq 0\right\}$ is finite. $H$ is a regressive function so by Fodor's theorem [2][8.7], there exists a $\mu<\omega_{1}$ such that $\left|H^{-1}\{\mu\}\right|=X_{1} . T_{\mu}^{*}$ is countable so by $(8)$ there is an $h \in T_{\mu}^{*}$ such that for each $\delta$, if $\mu<\delta<\omega_{1}$, then $h \cup\{(k, 0): \mu<k \leqslant \delta\} \in T^{*}$. It follows thus that

$$
\left\{h \cup\{(k, 0): \mu<k \leqslant \delta\}: \mu<\delta<\omega_{1}\right\}
$$

is an ordered subset of $\left(T^{*},<^{*}\right)$ of order type $\omega_{1}$.

\section{REFERENCES}

[I] CC. Chang and H.J. Keisler Model Theory. Studies in Logic and Foundations of Mathematics. North Holland.

[2] Jech T., Set Theory. The third Millenuim Edition Revised and Expanded. Springer.

[3] J. Knight Prime and Atomic models Journal of Symbolic Logic vol 43, no 3, I978 p. 385-393

[4] M. Laskowski, S. Shelah On the existence of atomic models Journal of Symbolic Logic, vol 58, no 4, I993, p. II89-II94 
The Australasian fournal of Logic (ISSN I448-5052) disseminates articles that significantly advance the study of logic, in its mathematical, philosophical or computational guises. The scope of the journal includes all areas of logic, both pure and applied to topics in philosophy, mathematics, computation, linguistics and the other sciences.

Articles appearing in the journal have been carefully and critically refereed under the responsibility of members of the Editorial Board. Only papers judged to be both significant and excellent are accepted for publication.

The journal is freely available at the journal website at

$$
\text { http://www.philosophy.unimelb.edu.au/ajl/ }
$$

All issues of the journal are archived electronically at the journal website.

SuBSCRIPTIONS Individuals may subscribe to the journal by sending an email, including a full name, an institutional affiliation and an email address to the managing editor at ajl-editors@unimelb.edu.au Subscribers will receive email abstracts of accepted papers to an address of their choice. For institutional subscription, please email the managing editor at ajl-editors@unimelb.edu.au.

Complete published papers may be downloaded at the journal's website at http: //www.philosophy.unimelb.edu.au/ajl/ The journal currently publishes in pdf format.

Submission The journal accepts submissions of papers electronically. To submit an article for publication, send the $\mathrm{LT}_{\mathrm{E}} \mathrm{X}$ source of a submission to a member of the editorial board. For a current list of the editorial board, consult the website.

The copyright of each article remains with the author or authors of that article. 